\title{
Inhalt des 3. Bandes
}

BRUNE, H., Zur Beeinflussung der Resorption von Wirkstoffen durch Adsorbentien . . I

ORTH, A. und F. монR, Fütterungsversuche an Jungrindern mit stark Blausäure abspaltenden Leinrückständen . . . . . . . . . . . 31

POLHEIM, P. voN, und H. Kummer, Die künstlich ermittelte Verdaulichkeit des Rohproteins von nordbadischem Wiesenheu im Vergleich zu den Verdauungskoeffizienten der Futterwerttabellen ............ 40

NICKISCH, к., Uber die Verdaulichkeit von Sonnenblumensamen und Sonnenblumenextraktionsschrot beim Kaninchen und Wiederkäuer . . . . . . . . $4^{8}$

schUlz, A., Uber den Fluorstoffwechsel bei Kaninchen und weitere Ausarbeitung des Analysenganges . . . . . . . . . . . . . .

wrTT, M., Untersuchungen über den Einfluß von Palmkern- und Kokoskuchen sowie Palmkern- und Kokosschroten mit unterschiedlichem Fettgehalt auf Milchmenge und Fettgehalt der Milch . . . . . . . . . . . . . 80

Nehring, $\boldsymbol{k}$. und w. Schramm $\dagger$, Zusammensetzung und Futterwert von Abfallfutterstoffen aus dem Gemüsebau ... . . . . . . . . . . . I02

SCHARPENSEel, H. W., Die Vitamingehalte der Rauhfuttermittel (Luzerne-, Rotklee und Wiesenheu) in Abhängigkeit von Werbungsart, Mineralstoffaufnahme und Witterung während des Trocknens . . . . . . . . . . . . 122

SCharpenseet, H.w., Vergleichende Untersuchungen zur Rohfaserbestimmung und Ermittlung des Produktionswertes von Rauhfuttermitteln . . . . . . . . I37

WENIGER, J. und K. FUNK, Ausschlachtungsversuche und Schlachtmethoden an Schweinen unter Berücksichtigung ihrer Futterverwertung . . . . . . . . I45

SChrader, K., R. SChreiber und K. Kt'Hn, Uber die Zusammenhänge zwischen dem Entwicklungszusțand der Luzerne und ihrer Silierfähigkeit . . . . . . . . . 160

SCHRAdER, k., R. SCHREIBER und H. KÜHN, Silierversuche über die Nährstoffverluste beim Anwelkverfahren . . . . . . . . . . . . . . 177

MUNDT, wolfram, Tëerarzt Dr. med. vet., Die Bedeutung der Darmflora unserer Haustiere unter besonderer Berücksichtigung einer Zugabe von Antibioticis in das Futter . . . . . . . . . . . . . ${ }^{188}$

ENGLERT, н. K., H. BAUER und H. KUMMER, Uber die Fischmehlkrankheit des Schweines i95

wUSSow, w., J. H. WENiger und H. R. SChUMm, Die Wirkung einer APF-Beigabe auf Futterverwertung und Schlachtleistung bei Schweinen . . . . . . . 223

DREYER, HEINZ, Untersuchungen über den Einfluß von Vitamin $B_{12}$ und APF auf den Eiweißstoffwechsel . . . . . . . . . . . . . . . . 240

havermanN, H. und H. ScharpenseEL, Untersuchungen über das Verhalten des d, E-Methionins im Stoffwechsel des vollwertig und nicht vollwertig ernährten Schweins und Legehuhns ..................2 
BRUNE, H., Fehlernährung mit Rübenblattsilage und Troblako ........ 281

DEGENER, w., Betrachtungen über den nutritiven Wirkungsmechanismus von Antibiotika ..................... 31 3

wENIGER, э. H. und K. FUNK, Untersuchungen über den Calcium- und Phosphorgehalt ganzer Schweinekörper .............. 325

SCHILLER, KLÄRE, Über den Ergänzungswert von Eiweißträgern verschiedener Herkunft in der tierischen Ernährung . . . . . . . . . . . . 342

STAMPA, s., Ein Beitrag zur Futterverwertung mit kritischer Verarbeitung bisheriger Versuchsergebnisse ............... $36 \mathrm{r}$ 
